\title{
Aplicación de la metodología Comunica't a la mejora de la competencia Comunicación Oral Efectiva de los alumnos del Master de Gestión de la Seguridad Alimentaria y la Calidad de la UPV
}

\author{
Édgar Pérez-Esteve ${ }^{a}$, Joan Albert Silvestre-Cerdà ${ }^{b}$, Maria del Carmen Bas ${ }^{c}$, María \\ Jesús Lerma-García ${ }^{d}$, Nuria Matarredona-Desantes ${ }^{\mathrm{e}}$ y Raúl Oltra-Badenes ${ }^{\mathrm{f}}$
}

aDepartamento de Tecnología de Alimentos, Universitat Politècnica de València, edpees@upv.es ; bepartamento de Sistemas de Información y Computación, Universitat Politècnica de València, jsilvestre@dsic.upv.es; 'Departamento de Matemáticas para la Economía y Empresa, Universitat de València, maria.c.bas@uv.es; dDepartamento de Química Analítica, Universitat de València, m.jesus.lerma@uv.es; 'Departamento de Composición Arquitectónica, Universitat Politècnica de València, numade@upvnet.upv.es; fDepartamento de Organización de Empresas, Universitat Politècnica de València, rauloltra@doe.upv.es

\begin{abstract}
Developing the "Effective Oral Communication" student outcome is a new strategy within the university studies, both in Bachelor's and Master's degrees. To reach this goal, the Comunica't methodology was created in 2017. This methodology has been developed within a Project of Innovation and Educational Improvement (PIME) at the Polytechnic University of Valencia, and has as its fundamental objective that the student, after performing a self-diagnosis, can improve their effective oral communication through a self-learning itinerary. In this way, the student, starting from his level of initial proficiency in terms of effective oral communication, follows a self-formative itinerary particularized to his specific situation, with the appropriate training activities. After two academic years of implementation, the goal of this work is to analyse the degree of contribution of this methodology to development of the "Effective Oral Communication" students outcome of students that followed the methodology during the subject Audits of Food Safety and Quality Systems, belonging to the Master in Food Safety and Quality Management. Results showed that the methodology contributed in a great extent to improve the ordering of ideas in the presentation, the non-verbal communication and the design of the slides.
\end{abstract}

Keywords: Effective oral communication, student outcome, self-learning itinerary

\section{Resumen}

Desarrollar el resultado estudiantil de "Comunicación oral efectiva" es una nueva estrategia dentro de los estudios universitarios, tanto en licenciatura como en másteres. Para alcanzar este objetivo, se creó la metodología Comunica't en 2017 dentro de un Proyecto de Innovación y Mejora Educativa. (PIME) en la Universitat Poliècnica de València. El proyecto 
tiene como objetivo fundamental que el estudiante, después de realizar un test de autodiagnóstico, pueda mejorar su comunicación oral efectiva a través de un itinerario de autoaprendizaje. De esta manera, el estudiante, a partir de su nivel inicial de competencia en términos de comunicación oral efectiva, sigue un itinerario autoformativo particularizado a su situación especifica, con las actividades de capacitación apropiadas. Después de dos años académicos de implementación, el objetivo de este trabajo es analizar el grado de contribución de esta metodología al desarrollo de la competencia "Comunicación oral efectiva" de los estudiantes que siguieron la citada metodología durante la asignatura Auditorías de Sistemas de Gestión de la Calidad y Seguridad Alimentaria, perteneciente al Máster en Gestión de Calidad y Seguridad Alimentaria. Los resultados mostraron que la metodología contribuyó en gran medida a mejorar el orden de las ideas en la presentación, la comunicación no verbal y el diseño de las diapositivas.

Palabras clave: Comunicación oral efectiva, competencia transversal, itinerario de autoaprendizaje

\section{Introducción}

Desde la introducción de las competencias transversales de los estudiantes en los estudios universitarios, la "Comunicación efectiva" es una de las más trabajadas en los tres niveles de domino de la competencia (Grado -1 y 2- y Master -3-). A pesar de su importancia, la disparidad en el dominio por parte de los estudiantes hace que sea difícil trabajarla en el aula a través de actividades colectivas. Por lo tanto, en la mayoría de las asignaturas solo se evalúa, sin dar al alumno herramientas para desarrollarla. En este contexto, un grupo de profesores de la Universitat Politècnica de València (UPV) y de la Universitat de València (UV) propusimos una metodología para evaluar el nivel inicial de la competencia comunicación oral efectiva a través de una herramienta de autodiagnóstico y, con base al resultado de la misma, desarrollar un itinerario de autoaprendizaje para los estudiantes incluyendo actividades de aprendizaje dirigidas a mejorar su dominio de la competencia (Oltra-Badenes et al., 2018). Esta metodología fue creada en el contexto del Proyecto de Innovación y Mejora Educativa (PIME/2017/B/025-14), financiado por la Universitat Politècnica de València.

A lo largo del proyecto, se han llevado a cabo diferentes acciones: a) diseño de una prueba de autoevaluación sobre la competencia comunicación efectiva (Lerma-García et al., 2018), b) desarrollo de un itinerario autoformativo basado en el nivel inicial de competencia (Pérez-Esteve et al., 2018) y c) evaluación del impacto de la metodología en diferentes grupos de estudiantes.

En una primera experiencia, la metodología se probó con estudiantes de primer año del grado en arquitectura (nivel de dominio I) (Bas et al., 2018). Los resultados de esta primera experiencia han revelado que la metodología propuesta no requiere mucho tiempo de clase, 
ya que es un aprendizaje autónomo por parte del estudiante aunque, al mismo tiempo, supervisado por el profesor para cumplir con un contrato de aprendizaje establecido por ambas partes. Los resultados de estas primeras experiencias también han demostrado que los estudiantes mejoraron significativamente su nivel de dominio.

\section{Objetivo}

El objetivo de esta segunda prueba piloto es evaluar la eficiencia de la metodología Comunica't con un grupo de estudiantes de la asignatura Auditorías de Sistemas de Gestión de la Calidad y Seguridad Alimentaria, perteneciente al Máster en Gestión de Calidad y Seguridad Alimentaria de la Universitat Politècnica de València, y por tanto de nivel de dominio III (Master) y determinar: a) la contribución de la metodología a la mejora de la competencia Comunicación Oral Efectiva y b) satisfacción de los alumnos con la metodología propuesta.

\section{Desarrollo de la innovación}

\subsection{Descripción y contexto de la asignatura "Auditorías de Sistemas de Gestión de la Calidad y Seguridad Alimentaria"}

La asignatura "Auditorías de la calidad de los alimentos" pertenece al Máster Universitario en Seguridad y Gestión de la Calidad de los Alimentos que ofrece la Universitat Politècnica de València. El objetivo de la asignatura es diseñar planes de auditoría para evaluar la calidad y la seguridad de los alimentos, así como desarrollar las competencias básicas de un auditor. En el curso académico 2017-2018, la asignatura fue seguida por 32 alumnos, de los cuales 30 participaron en esta experiencia.

\subsection{Metodología Comunica't.}

La metodología Cumunica't se aplicó a este estudio de la siguiente manera. En primer lugar se pidió a los estudiantes que prepararan una breve presentación de un tema relacionado con los sistemas de gestión de la calidad (Normas ISO). Durante la presentación, los estudiantes fueron evaluados tanto por sus compañeros de clase, como por los profesores, con la ayuda de una rúbrica digital alojada en un servidor web. Después de las presentaciones, los profesores recopilaron las puntuaciones y todos los comentarios de los compañeros de clase y elaboraron un informe individual para cada uno de los estudiantes. En segundo lugar se invitó a los alumnos a realizar una prueba de autoevaluación para ayudarlos a reflexionar sobre sus puntos débiles y fuertes en la preparación y ejecución de la presentación oral (Lerma-García et al., 2018). En base a la puntuación obtenida en cada uno de los apartados del test de autoevaluación, se recomendó a los estudiantes trabajar en actividades específicas para reforzar sus debilidades (Pérez-Esteve et al., 2018). Al final del curso, se pidió a los estudiantes que prepararan una nueva presentación, que fue evaluada nuevamente por sus compañeros de clase y por los profesores de la asignatura, utilizando la 
misma rúbrica que al inicio. Después de todo el proceso, los estudiantes tuvieron que preparar un portafolio que reuniera todas las reflexiones sobre sus debilidades, fortalezas y cómo las diferentes actividades propuestas habían contribuido a mejorar sus habilidades de comunicación.

La metodología queda resumida en la figura 1.

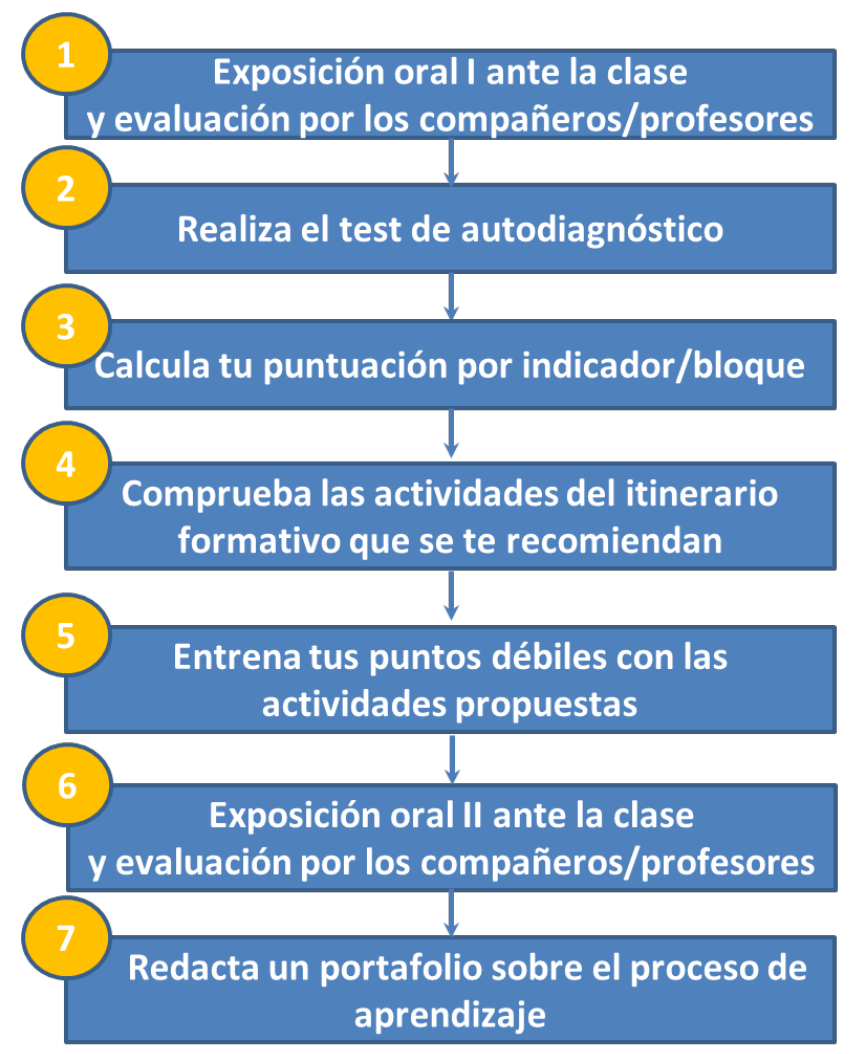

Fig. 1 Esquema de la metodología propuesta

\subsection{Evaluación de la contribución de la metodología a la mejora de la competencia Comunicación Oral Efectiva}

Para evaluar la eficiencia de la metodología propuesta, se siguieron diferentes enfoques: una comparación de las notas obtenidas tras la exposición I y II por parte de los profesores, la evaluación de su portafolio de aprendizaje y una encuesta de satisfacción contestada de manera electrónica al acabar la asignatura. La prueba de satisfacción incluyó 12 preguntas a través de las cuales se evaluó la opinión de los estudiantes con la metodología. Se usó una escala de cinco puntos de "totalmente de acuerdo" (calificada como 5) a "totalmente en desacuerdo" (calificada como 1) para determinar el nivel de acuerdo con cada declaración. Las encuestas se realizaron a través de Poliformat, una plataforma de enseñanza desarrollada por la Universitat Politècnica de València. 


\section{Resultados}

\subsection{Nivel de dominio de la competencia antes de la intervención}

La Figura 2 muestra los porcentajes de estudiantes que durante la segunda exposición de la materia obtuvieron una calificación de A (excelente), B (muy buena), C (aceptable) o D (criterio no alcanzado) en cada uno de los ítems evaluados: dominio del tema, organización del contenido, lenguaje verbal, lenguaje no verbal, herramientas de presentación (básicamente Microsoft Power Point) y elaboración de respuestas a las preguntas de los profesores.

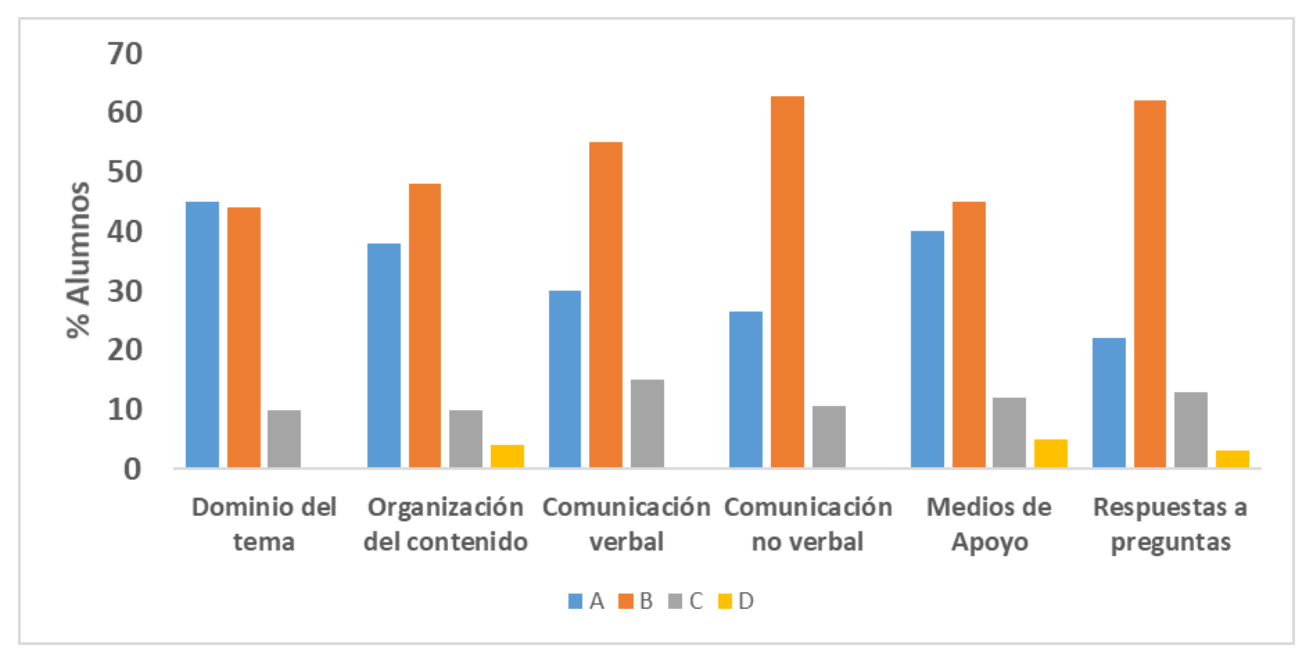

Figura 2. Puntuación obtenida por los estudiantes en las diferentes áreas de dominio del resultado de la comunicación efectiva, expresado como un porcentaje de estudiantes calificados con las letras $A-D$.

En la figura se observa que los alumnos presentaron un buen dominio del tema, superando los alumnos con A el porcentaje de alumnos con B, C y D. La segunda área de dominio donde los estudiantes fueron más competentes fue el de utilización de medios de apoyo, pero esta vez el porcentaje de alumnos con puntuación B fue el predominante. Los fallos principales en esta área fueron el uso de plantillas muy recargadas que no resaltan las ideas principales, el uso de animaciones y transiciones innecesarias, el uso de tamaños de letra muy pequeños y el abuso del texto. La comunicación verbal fue la tercer área en orden de dominio. Los peores resultados se obtuvieron en comunicación verbal (30\% de los alumnos con A), respuesta a preguntas ( $30 \%$ de los alumnos con A) y comunicación no verbal $(27 \%$ de los alumnos con A). En estos casos, los errores más comunes fueron utilizar un vocabulario genérico no propio del área de conocimiento, el responder a aspectos diferentes a los que se preguntaba, el uso excesivo de coletillas, la posición estática y el uso excesivo de muletillas. 


\subsection{Nivel de dominio de la competencia tras la intervención}

La Figura 3 muestra los porcentajes de estudiantes que durante la segunda exposición de la materia obtuvieron una calificación de A (excelente), B (muy buena), C (pase) o D (criterio no alcanzado) en cada uno de los ítems evaluados: dominio del tema, organización del contenido, lenguaje verbal, lenguaje no verbal, herramientas de presentación (básicamente power point) y elaboración de respuestas a las preguntas de los profesores.

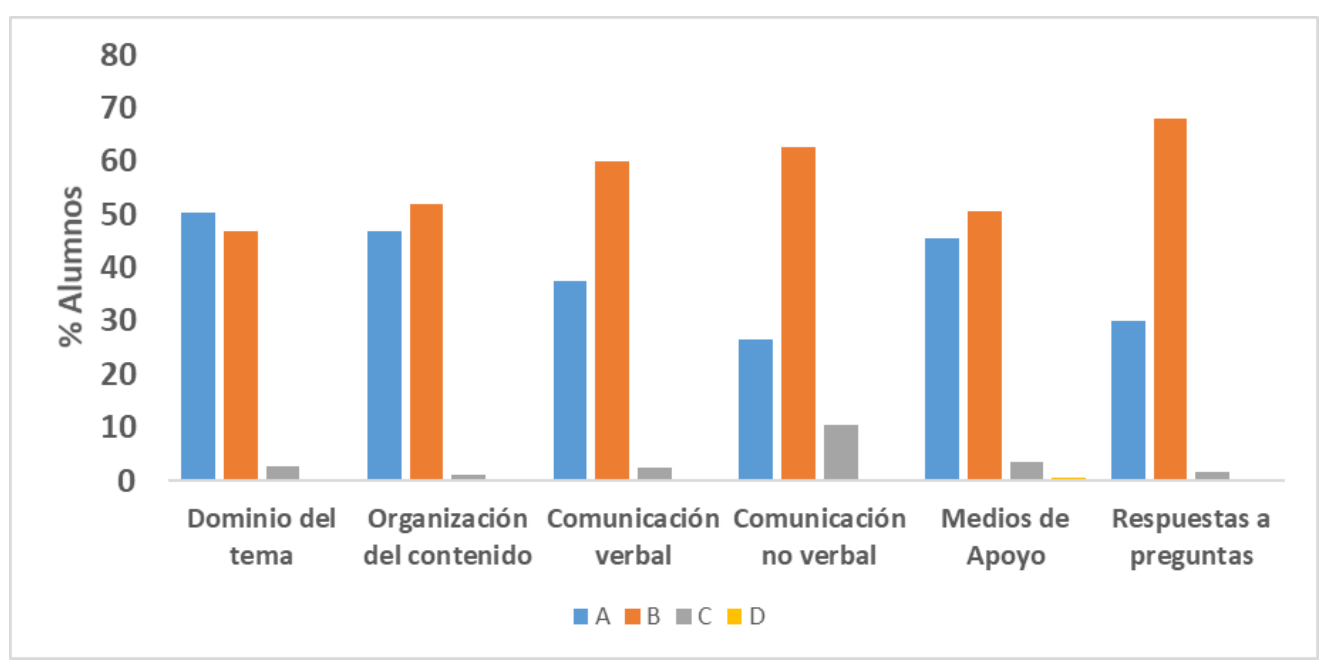

Figura 3. Puntuación obtenida por los estudiantes en las diferentes áreas de dominio del resultado de la comunicación efectiva, expresado como un porcentaje de estudiantes calificados con las letras $A-D$.

Como puede verse en la figura, casi el 100\% de los estudiantes recibió una calificación de A o B en la mayoría de los parámetros calificados. También se puede observar cómo el dominio del tema, la organización del contenido y el uso de la herramienta de presentación fueron las áreas de competencia donde las calificaciones fueron más altas. En contraste, los estudiantes recibieron calificaciones más bajas en la comunicación no verbal y su capacidad para responder adecuadamente a las preguntas formuladas.

La comparación de las figuras 2 y 3 permite evidenciar que, tras la intervención propuesta, el porcentaje de alumnos con A aumentó en todas las áreas de dominio al mismo tiempo que disminuyó el número de alumnos puntuados con B. Por otra parte, desapararecioron las calificaciones con D.

De acuerdo con estos resultados, la metodología ha contribuido mucho al desarrollo del resultado efectivo de la comunicación del estudiante. Sin embargo, hay áreas de dominio, en las que las actividades han sido menos efectivas para garantizar que más del $50 \%$ de los estudiantes obtuvieron una calificación excelente (A).

\subsection{Satisfacción de los alumnos con la metodología propuesta}

Para conocer el grado de satisfacción de los estudiantes con la metodología, al final de la asignatura, los estudiantes recibieron una encuesta de satisfacción con la metodología via 
mail para ser contestada electrónicamente. La Figura 4 muestra el porcentaje de estudiantes que concedieron cada una de las diferentes puntuaciones entre 1 (totalmente en desacuerdo) y 5 (totalmente de acuerdo). Como se puede observar, el $85 \%$ de los estudiantes estuvieron de acuerdo en que las preguntas de la prueba de autoevaluación se formularon de manera que podían entenderse fácilmente (Fig. 4.a). La Figura 4.f muestra que la mayoría de los estudiantes tomaron de 8 a 12 minutos para completar la prueba de autoevaluación. A pesar de esta inversión de tiempo, el 90\% de los estudiantes estuvo de acuerdo en que este tiempo era adecuado (Fig. 4.b). Los estudiantes también evaluaron la simplicidad del procedimiento para calcular su nivel de competencia. La Figura 4.c muestra que el 70\% de los estudiantes estuvo de acuerdo en que el procedimiento fue simple. Sin embargo, podría mejorarse utilizando una calculadora automática.

Del mismo modo, se le preguntó sobre la facilidad de seleccionar las actividades que debían realizar durante la fase de autoaprendizaje en función de sus resultados. Nuevamente, el $70 \%$ de los encuestados respondió que era fácil saber qué actividades deberían llevarse a cabo. Sin embargo, el uso de una aplicación web que hiciera el cálculo del nivel de competencia y propusiera las actividades de manera automática simplificaría enormemente el proceso.

La Figura 4.e muestra la satisfacción de los estudiantes con las actividades incluidas en el itinerario de autoaprendizaje. Como se puede observar, el nivel de satisfacción no varió significativamente en función del bloque de actividades. También se puede observar que el $70-75 \%$ de los encuestados estaban satisfechos o realmente satisfechos con las actividades propuestas, y que más del $92 \%$ de los encuestados estaban satisfechos o realmente satisfechos con las actividades en su conjunto.

Finalmente, la Figura 4.g muestra la satisfacción global de los estudiantes con la metodología. Como puede verse, el $75 \%$ de los estudiantes estaban satisfechos o realmente satisfechos, a pesar del hecho de que habían invertido mucho tiempo en realizar las tareas propuestas: preparar una presentación, presentar el tema frente a los compañeros, realizar un test de autoevaluación, completar diferentes tareas y preparar y exponer una nueva presentación. 

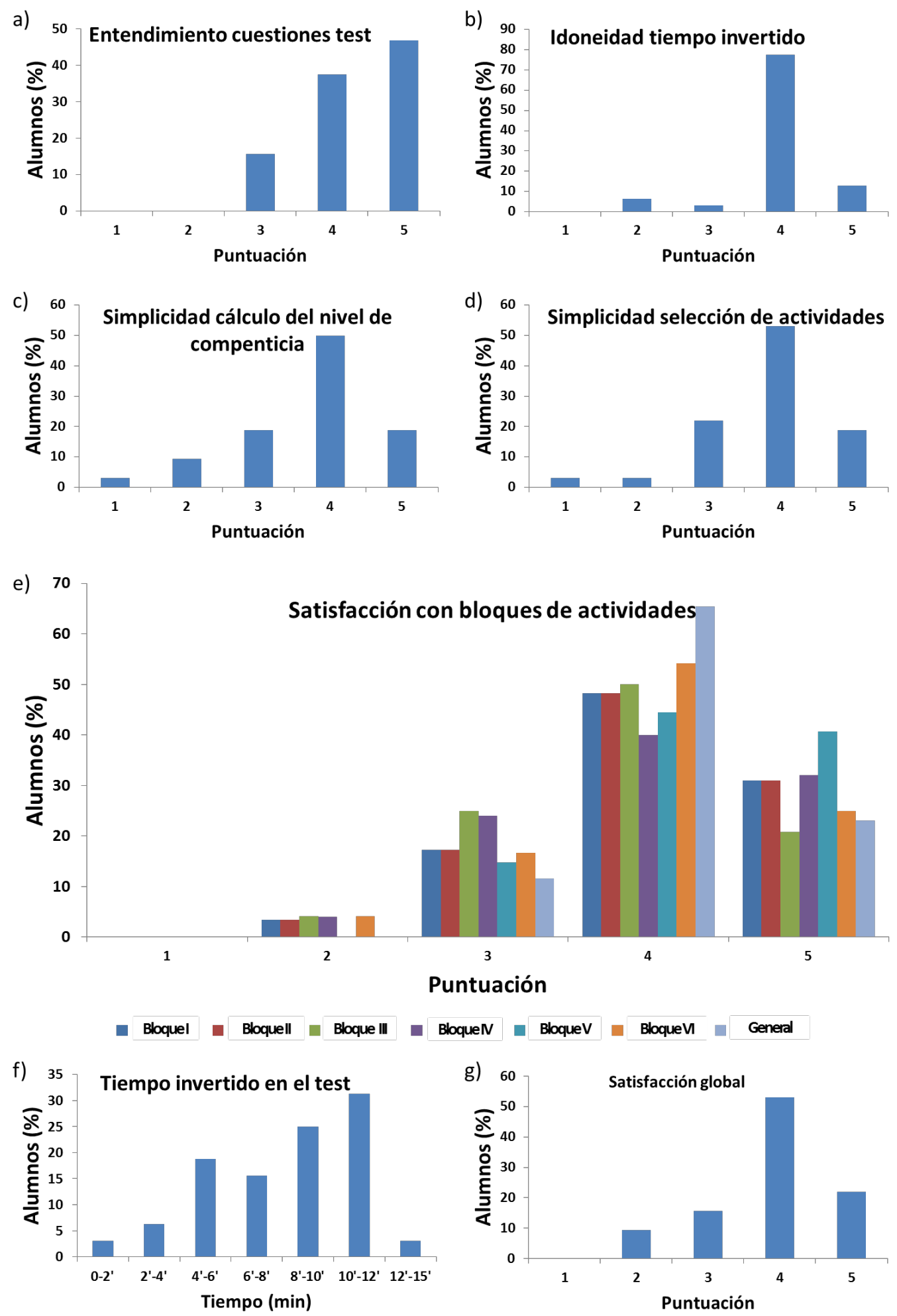

Figura 4. Resultados de la encuesta de satisfacción.. 


\section{Conclusiones}

La metodología propuesta basada en la autoevaluación y el autoaprendizaje requiere un esfuerzo significativo en términos de tiempo por parte de los estudiantes. Sin embargo, aunque hay campos en los que la metodología contribuyó a un porcentaje más bajo, en base a los resultados académicos globales y los resultados de la encuesta de satisfacción, se puede concluir que la metodología contribuye exitosamente al desarrollo de la competencia de comunicación efectiva de los estudiantes. Para facilitar aún más el desarrollo de la competencia, los estudiantes proponen la creación una aplicación web que integre todas las fases de la metodología.

\section{Referencias}

OLTRA-BADENES R, PEREZ-ESTEVE E, SILVESTRE-CERDA JA, BAS MC, LERMA-GARCIA M.J, MATARREDONA-DESANTES N (2018). "PIMECOE: A project for self-diagnosis of the sudent outcome "effective communication" and its improvement through a self-formative itinerary" en INTED Proceedings, p. 3800 - 3805.

LERMA-GARCIA MJ, MATARREDONA-DESANTES N, OLTRA-BADENES R, PEREZESTEVE E, SILVESTRE-CERDA JA, BAS MC (2018) "Development of a self-diagnosis guide to assess the initial proficiency level of the student outcome "Effective oral communication" en EDULEARN Proceedings, p. 4020 - 4025

PEREZ-ESTEVE E, SILVESTRE-CERDA JA, BAS MC, LERMA-GARCIA MJ, MATARREDONA-DESANTES N, OLTRA-BADENES R (2018) "Activities to promote the selflearning of the effective communication student outcome" en INTED2018 Proceedings, p. 38063812 .

BAS MC, LERMA-GARCIA MJ, MATARREDONA-DESANTES N, OLTRA-BADENES R, PEREZ-ESTEVE E, SILVESTRE-CERDA JA (2018) "Self learning itinerary as a tool to improve effective communication: a qualitative analysis of a pilot experience" en EDULEARN Proceedings, p. $4049-4056$. 